\title{
ACCIONES DE RESPONSABILIDAD SOCIAL MERCADOLOGICA EN TORNO AL COVID-19 EN RESTAURANTES DE LA CIUDAD DE CHILPANCINGO, GUERRERO
}

\author{
MARKETING SOCIAL RESPONSIBILITY ACTIONS AROUND COVID-19 IN \\ RESTAURANTS IN THE CITY OF CHILPANCINGO, GUERRERO
}

\author{
José Luis Susano-García $^{1}$ * (D); María del-Socorro Cabrera-Ríos ${ }^{2}$ (iD; Martha Adela Bonilla-Gómez ${ }^{2}$ (iD). \\ 1. Universidad Autónoma de Guerrero, México.jose.susano@uagro.mx \\ 2. Universidad Autónoma de Guerrero, México. \\ *Autor de Correspondencia: José Luis Susano-García, correo electrónico: jose.susano@uagro.mx
}

\section{RESUMEN}

Derivado de la contingencia sanitaria se ha generado una serie de problemáticas que ha afectado de manera directa a las empresas, en ese sentido las afectaciones en materia de mercadotecnia, son bajas ventas, canales de distribución, nuevas formas de consumo, uso de tecnologías, nuevas necesidades entre otras; que se reflejan en el posicionamiento y competitividad de las empresas.

El trabajo versa sobre un estudio exploratorio realizado a MiPYMES del sector servicios, caso concreto restaurantes de la ciudad de Chilpancingo, Guerrero, con la finalidad de conocer las acciones de Responsabilidad Social mercadológica que han aplicado para hacer frente a la pandemia del COVID-19 y cuidar la salud de sus consumidores y empleados.

Entre los hallazgos encontrados en el estudio referentes a la salud de los consumidores y empleados de los restaurantes están reducción de número de comensales, separación de espacios, colocación de tapete sanitizante, gel antibacterial, medidor de temperatura, toma de pedidos por internet, redes sociales, ventas telefónicas, cobros por tarjeta y depósito bancario, kit de protección para trabajadores, comunicación de información en materia de salud, etc.

Palabras claves: Responsabilidad Social; mercadotecnia; consumidores; restaurantes.

Cómo citar:

Susano-García, José Luis; Cabrera-Ríos, María del-Socorro; Bonilla-Gómez, Martha Adela. (2021). Acciones de responsabilidad social mercadologica en torno al Covid-19 en restaurantes de la ciudad de Chilpancingo, Guerrero. Revista de Investigaciones Universidad del Quindio, 33(S1), 240-250. https://doi.org/10.33975/riuq.vol33nS1.496 


\begin{abstract}
Derived from the health contingency, a series of problems have been generated that have directly affected companies, in that sense the effects on marketing are low sales, distribution channels, new forms of consumption, use of technologies, new needs among others; which are reflected in the positioning and competitiveness of companies.

The work is about an exploratory study carried out to MSMEs in the service sector, a specific case of restaurants in the city of Chilpancingo, Guerrero, in order to know the marketing Social Responsibility actions that have been applied to face the COVID-19 pandemic and take care of the health of its consumers and employees.

Among the findings found in the study regarding the health of consumers and restaurant employees are a reduction in the number of diners, space separation, placement of a sanitizing mat, antibacterial gel, temperature meter, taking orders online, social networks , telephone sales, card charges and bank deposit, protection kit for workers, communication of health information, etc.
\end{abstract}

Keywords: Social Responsibility, marketing, consumers, restaurants.

\title{
INTRODUCCIÓN
}

En la actualidad las empresas sean de giro, tamaño y sector que sean no esperaban que los efectos de la pandemia por el COVID-19 generara diversas problemáticas, por mencionar algunas están las bajas ventas, posicionamiento de las empresas, baja competitividad, el cierre o en el peor de los casos el quiebre de los negocios.

En este sentido los consumidores al estar ávidos de adquirir productos y servicios que satisfaga sus necesidades, siempre y cuando las empresas cuiden los aspectos de salud de forma responsable.

Es por ello que el trabajo versa en realizar un estudio que muestre indicadores, los cuales se relacionen con aspectos de responsabilidad social en torno al cuidado de la salud de los consumidores y sus empleados, caso concreto de los restaurantes de la ciudad de Chilpancingo, Guerrero.

Respecto a la clasificación y características de las unidades de negocios en México, el INEGI (2018), la MiPYMES se estratifican de la siguiente manera:

Tabla 1. Estratificación de las empresas por sector económico

\begin{tabular}{llll}
\hline \multirow{2}{*}{ Tamaño de empresa } & \multicolumn{4}{l}{ Número de personas ocupadas } \\
\cline { 2 - 4 } Micro & Manufactura & Comercio & Servicios \\
\hline Pequeña & $1-10$ & $1-10$ & $1-10$ \\
\hline Mediana & $11-50$ & $11-30$ & $11-50$ \\
\hline
\end{tabular}

Fuente: INEGI (2020)

Existen diversas situaciones que afectan de manera directa e indirecta a las empresas, factores como desarrollo de la tecnología, la globalización, cuestiones económicas, situaciones ambientales, aspectos políticos, situaciones de salud, entre otros. Por ejemplo, en la actualidad la pandemia por el COVID-19 
ha puesto en jaque a todo el sector económico a nivel mundial, en este sentido las empresas, negocios, productos, marcas y servicios.

El motivo de realizar este estudio versa desde la importancia que tienen las MiPYMES en México, pues de acuerdo a datos del INEGI (2020) el 93.4\% son microempresas, 5.9\% Pequeñas y Medianas y el $0.7 \%$ grandes empresas, del total de las microempresas. Por lo anterior, se consideraron a los restaurantes que existen en la ciudad para aplicar el estudio, debido a que son medianas empresas que cuentan con información relevante y son un motor importante en la economía local. (Susano, et al. 2020).

López (2018), menciona que los restaurantes al ser unidades de negocios entran en una clasificación de PYMES las cuales presentan algunas problemáticas que impiden su competitividad, es por ello la importancia también de realizar este estudio para conocer algún dato que se relacione entre la Responsabilidad Social y la competitividad.

Ramírez, M. (2021), menciona que "es importante conocer la situación en torno a la pandemia en la cual se encuentran los empresarios debido a la importancia que tiene el sector de preparación de alimentos, tanto por el número de establecimientos como por el número de empleos que general, se ha decidido llevar a cabo esta investigación en el sector restaurantero, específicamente la forma de como comercializan su producto".

Resulta significativo mencionar en el caso de la ciudad de Chilpancingo, al depender en su mayoría del comercio y los servicios, la economía se vio estancada al haber cierres y riesgos de quiebre en varios establecimientos comerciales y de servicios, estos últimos como hoteles y restaurante, es por ello la importancia de identificar las acciones en materia de responsabilidad social que el sector restaurantero ha implementado a fin de responder de manera responsable con acciones encaminadas al cuidado de la salud de sus consumidores.

En este sentido, una de las necesidades más importantes tiene que ver con la necesidad primaria que es la alimentación y para ello, en la ciudad de Chilpancingo existen diferentes establecimientos y categorías, como lo son restaurantes, cocinas económicas, establecimientos de comida rápida, puestos ambulantes, entre otros.

Por lo anterior el presente trabajo busca identificar cuáles son las acciones que en materia de Responsabilidad Social las empresas restauranteras de la ciudad de Chilpancingo han implementado con la finalidad de cuidar la salud tanto para su consumidores y empleados.

\section{MARCO TEÓRICO}

\section{Importancia de la Responsabilidad Social en los negocios}

Sin duda alguna la Responsabilidad Social es una acción importante para los negocios, en este sentido de acuerdo a Añies y Bonomie (2010), indican que la Responsabilidad Social en los negocios implican políticas, prácticas y programas incorporadas a las estrategias empresariales, mismas que deben de resolver problemáticas sociales.

Primeramente, se aborda la definición del concepto de Responsabilidad Social, de acuerdo a la Norma ISO 26000 (2010) que menciona que: 
"...la responsabilidad de una organización ante los impactos que sus decisiones y actividades ocasionan en la sociedad y el medio ambiente, a través de un comportamiento transparente y ético que: contribuya al desarrollo sostenible, incluyendo la salud y el bienestar de la sociedad; tome en consideración las expectativas de sus partes interesadas; cumpla con la legislación aplicable y sea coherente con las normas internacionales de comportamiento; y esté integrada en toda la organización y se lleve a la práctica en sus relaciones"

Sin embargo, para identificar a las acciones de la Responsabilidad Social y tener una claridad respecto a ellas, Secchi (2007) clasifica las teorías de la Responsabilidad Social de la Empresa [RSE] en tres grupos: utilitarias, relacionales y gerenciales.

Stanley (2014), menciona que existen seis dimensiones relacionadas con la Responsabilidad Social y el Marketing

1. Calidad de bienes y servicios, considerando cuestiones ambientales;

2. Calidad del servicio, facilitando las decisiones de compra;

3. Publicidad y prácticas de promoción, asociadas con la transparencia, veracidad y honestidad;

4. Información vinculada a propiedades, riesgos y limitaciones de productos y servicios;

5. Respeto a la privacidad;

6. Ética y compromiso social, relacionado a un consumo razonable y que no dañe al medioambiente.

Por otra parte, para Barragán, Guerra y Villalpando (2017), se deben de identificar las tendencias y requerimientos del mercado, para que estas sean integradas a las estrategias de mercadotecnia y de Responsabilidad Social, mismas que deben incidir en el modelo de negocios a través de seis actividades básicas, las cuales son:

1. Promover la calidad absoluta en bienes y servicios

2. Servicio al cliente que se traduzca en una experiencia positiva y de satisfacción al cliente

3. Publicidad y promoción sustentadas en la honestidad y veracidad

4. Información de transparencia al mercado

5. Respeto a la privacidad del consumidor

6. Ética y compromiso social

Es por ello que la aplicación o implementación de acciones de Responsabilidad Social debe tener un impacto positivo en la empresa, así lo indica Chang (2011), al considerar que existen efectos positivos cuando una empresa o marca tiene un comportamiento responsable, lo que se refleja en el posicionamiento y por lo consiguiente en la preferencia del consumidor.

Al ser considerada ya la Responsabilidad Social como una filosofía y no una moda que todas las empresas de los tamaños y giros que sean deben de implementarla, es necesario identificar sus acciones acorde a sus necesidades y alcances, en este sentido (Willer y Lernoud, 2016), hacen referencia a que existen productos y servicios éticos y socialmente responsables, mismos que se ofertan desde tiendas minoristas hasta grandes cadenas de supermercados.

Por otra parte derivado del COVID-19, el comportamiento del consumidor ha cambiado desde diferentes aspectos como son: búsqueda de información, adquisición de productos y servicios, formas de consumo, nuevas formas de relación con las marcas y empresas, entre otros. 
Por lo anterior Nielsen (2015) menciona que cuatro de cada diez consumidores pagarían más por productos de empresas socialmente comprometidas, en este sentido los restaurantes de la ciudad de Chilpancingo realizan ciertas acciones y desconocen realmente el sentir de los consumidores al preferirlos considerándolos como responsables al cuidar la salud.

Para Wong, et al (2020) afirman que actualmente los consumidores se están volviendo más conscientes socialmente y están incluyendo consideraciones éticas en sus decisiones de compra.

De igual forma existen diversas preferencias de los consumidores, mismas que se originan a partir de su comportamiento ético Esto se ha logrado a través de la gran diversidad de productos y, por lo tanto, existen más oportunidades de guiarse por sus preferencias racionales y éticas al momento de comprar, (Auger y Devinne, 2010).

Finalmente, la importancia de aplicar estrategias de responsabilidad social vinculadas con la mercadotecnia, tendrán como resultado el incremento de la competitividad de los negocios y ampliar el acceso a los mercados. Añies y Bonomie (2010).

\section{Problemas de las empresas en torno al COVID-19.}

Hablar de problemáticas de las unidades de negocios es referirse a diferentes aspectos, en este sentido, de acuerdo a de acuerdo a la ANPEC (2016), menciona que en el caso de las MiPYMES presentan diferentes problemas mismos que afectan su nivel de competitividad.

En otro contexto, Pineda, J. (2020) menciona que "la gran mayoría de las empresas de la región (estado de Guerrero) han registrado importantes caídas de sus ingresos y presentan dificultades para mantener sus actividades, ya que tienen serios problemas para cumplir con sus obligaciones salariales y financieras, y dificultades para acceder a financiamiento para capital de trabajo".

Pineda, J. (2020), indica que el impacto que ha tenido la pandemia ha generado de manera específica en las MiPYMES menores probabilidades de sobrevivencia y en algunos de los casos el cierre temporal o definitivo. Es por ello que actualmente de acuerdo a la situación actual que viven los negocios existe la necesidad de investigar a profundidad estas nuevas formas de mercadeo (Cortés, 2019).

CEPYME (2020) destaca que el 96\% de estas empresas afirman que se están viendo afectadas negativa o muy negativamente ante esta situación y un $67 \%$ considera que las expectativas generales en 2020 para su negocio son malas o muy malas, es por ello la urgente necesidad que tienen los negocios de todos los giros y tamaños para implementar estrategias innovadoras de mercadotecnia que le permitan hacer frente a esta situación actual para poder continuar en el mercado.

En México el INEGI (2020), en su encuesta del impacto económico generado por el COVID-19 menciona que del total de las empresas el 59.6\% instrumentó paros técnicos y temporales, por más de 21 días, mientras que el $40.4 \%$ de las microempresas cerraron, en la misma tesitura el organismo indica que el 93.2\% sufrió alguna de las siguientes afectaciones:

- Disminución de los ingresos

- Baja de demanda

- Escasez de insumos y/o productos 
- Reducción del personal

- Reducción de remuneraciones y/o prestaciones

Finalmente, Susano, et al. (2021), indican que "en la actualidad existen diferentes situaciones o problemáticas que afectan de manera directa e indirecta a las MiPYMES entre los factores están, la inseguridad, la situación social, la economía, los aspectos tecnológicos, la competencia, las tendencias de consumo y ahora en la actualidad, las cuestiones de salud, como el COVID-19”.

\section{MÉTODO}

El presente trabajo se aborda desde un tipo de investigación exploratoria y descriptiva, aplicada a los restaurantes de la ciudad de Chilpancingo, con la finalidad de conocer las acciones se relacionan con el cuidado y prevención de la salud, como una medida de Responsabilidad Social.

Respecto al universo de los servicios de restaurantes, se tomó de la información que existe en el Sistema de Información del INEGI (2020), en donde se indica que en la ciudad de Chilpancingo existen 45 restaurantes en las categorías de dos o más tenedores.

Para la determinación de la muestra a considerar se realizó un censo, es decir abarcar los 45 restaurantes ello con la finalidad de contar con toda la información respecto a sus acciones en materia de Responsabilidad Social ente el COVID-19.

Se realizó un esquema para determinar las variables y acciones que tuviesen relación con la RS.

\begin{tabular}{|c|c|c|}
\hline VARIABLE & \multicolumn{2}{|c|}{ ACCIONES } \\
\hline Plaza & $\begin{array}{l}\text { - Colocación de producto } \\
\text { - Separación de mesas } \\
\text { - Uso restringido de espacios } \\
\text { - Número de comensales }\end{array}$ & $\begin{array}{l}\text { - Código QR para carta } \\
\text { - Uso de Tableta } \\
\text { - Gel antibacterial, tapete sanitizante } \\
\text { y medidor de temperatura. }\end{array}$ \\
\hline Canal de distribución & - Entrega a domicilio & - Pedidos y entrega personal \\
\hline Comunicación & $\begin{array}{l}\text { - Mensajes de salud } \\
\text { - Información } \\
\text { procedimientos }\end{array}$ & $\begin{array}{l}\text { - Comunicación de horarios } \\
\text { - Redes Sociales }\end{array}$ \\
\hline Formas de pago & - Pago por tarjeta & - Depósito o transfer \\
\hline Personal & - Atención a clientes & - Kit de protección \\
\hline
\end{tabular}

Para la recolección de la información se utilizó la de encuesta como instrumento de recolección de datos, la forma de obtención de la información se llevó mediante la semaforización por parte de la Secretaria de Salud Estatal, para ello se trabajó en los días que marcaba el semáforo naranja, es decir del 2 al 10 de julio y del 9 al 15 de noviembre de 2020, dichas fechas sirvieron para la aplicación de las encuestas aplicadas a los dueños y administradores de los restaurantes, para ello se contactó de manera directa a los entrevistados y se generó una agenta a fin de concretar en tiempo y forma la aplicación de la encuesta.

Para el tratamiento de los resultados fueron tabulados en forma de gráficas descriptivas con la finalidad de dar una mejor información precisa al lector y con ellos evitar posibles malas interpretaciones. 


\section{RESULTADOS}

A continuación, se presentan a modo de gráfico descriptivo los resultados obtenidos, derivados de la encuesta realizada a los dueños y administradores de los diferentes restaurantes de la ciudad de Chilpancingo.

Gráfica 1. Acciones aplicadas por plaza

Acciones por Plaza

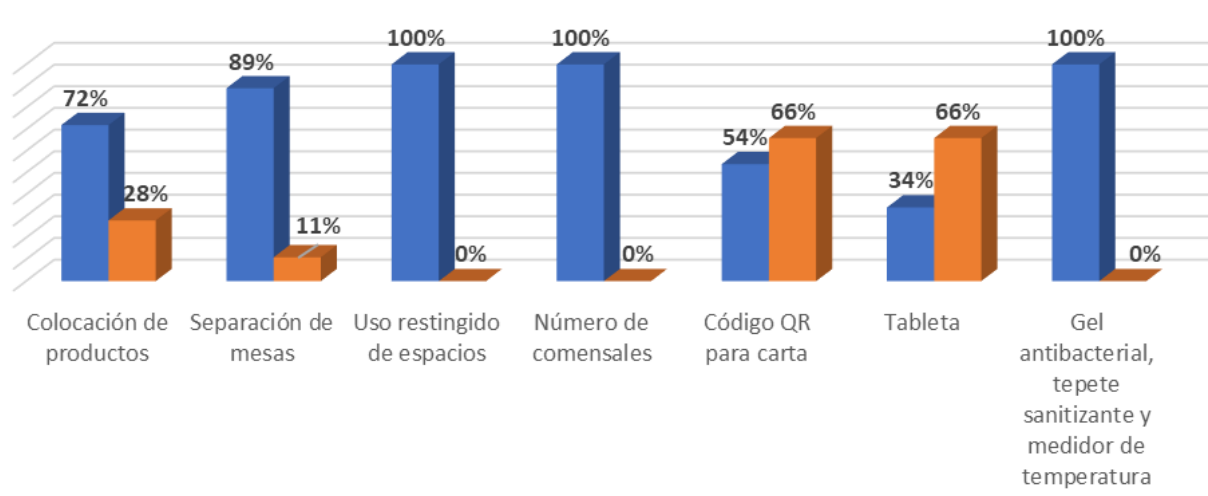

$\square \mathrm{SI}$ NO

Resulta significativo indicar que en los restaurantes se han aplicado en su mayoría acciones en torno a la Responsabilidad Social con el objetivo de llevar a cabo las indicaciones instruidas por autoridades de salud, en este sentido el 100\% ha utilizado el gel antibacterial, reducción en el número de comensales, uso restringido de espacios, seguido de la separación de mesas $(89 \%)$ y colocación de productos (72\%). En lo que respecta al uso de tecnología para mostrar la carta y hacer pedidos, estos por ser una inversión, son pocos los restaurantes que hacen uso de esta estrategia, mismos que se reflejan con más del $65 \%$ de los restaurantes que no hacen uso de la tecnología.

Gráfica 2. Acciones aplicadas por Canal de Distribución

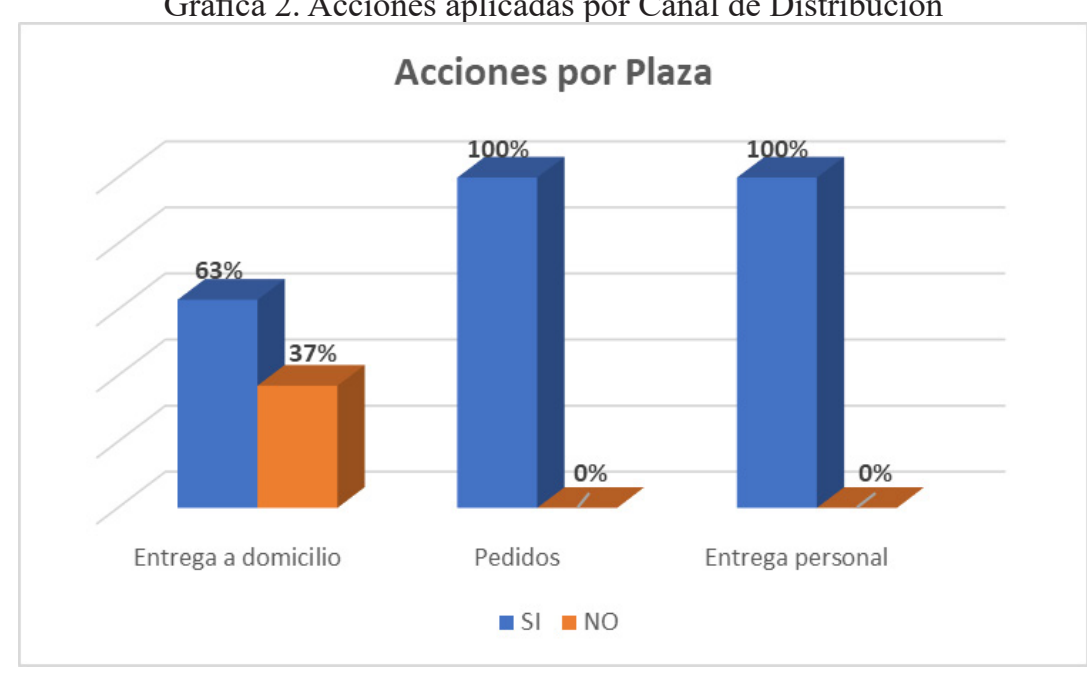

Hay dos acciones que los restaurantes hacen uso de ella mismas que impactan en la generación de no contactos físicos con los consumidores como parte de la Responsabilidad Social ante la pandemia, son las que corresponden a la realización de pedidos por teléfono, WhatsApp y por Facebook, estas estrategias son aplicadas por el 100\% de los restaurantes, ello debido a que la inversión es mínima. En lo que respecta a la entrega a domicilio el $63 \%$ de los restaurantes han optado por enviar los pedidos 
a los domicilios o centros de trabajo, ello de supone debido a que las empresas tienen que hacer una inversión ya sea en auto, gasolina, motocicleta, así como la contratación de una persona como repartidor.

Gráfica 3. Acciones aplicadas por Comunicación

\section{Acciones de Comunicación}

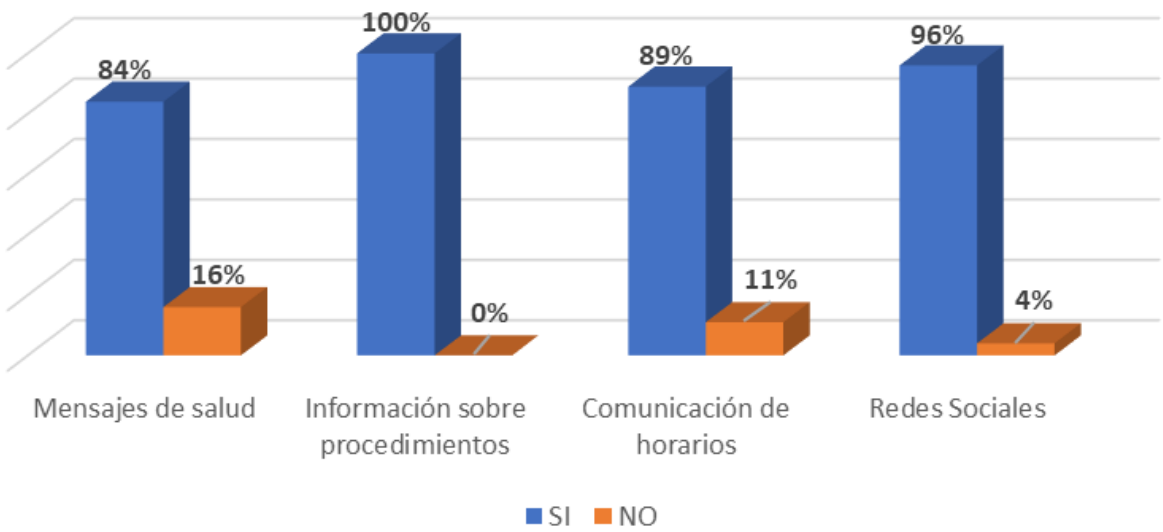

Como parte de la Responsabilidad Social los restaurantes implementaron acciones de comunicación con la finalidad de dar a conocer y difundir a través de diferentes formas los mensajes que la Secretaría de Salud estableció a fin de informar y comunicar a sus empleados y consumidores las formas de trabajo y prestación del servicio. Estas estrategias son de suma importancia, ello debido a que a través de la comunicación se emiten mensajes que informan y dan a conocer diferentes aspectos, en este sentido todos han mostrado una responsabilidad de comunicar procedimientos, el uso de redes sociales para diversas índoles como publicidad, realizar pedidos, comunicar promociones. De igual manera se han establecidos mensajes de salud impresos y electrónicos, así como la información de los horarios, los indicadores arrojas que todos las utilizan con un margen superior al $80 \%$.

Gráfica 4. Acciones aplicadas por Formas de Pago

\section{Acciones en Formas de Pago}

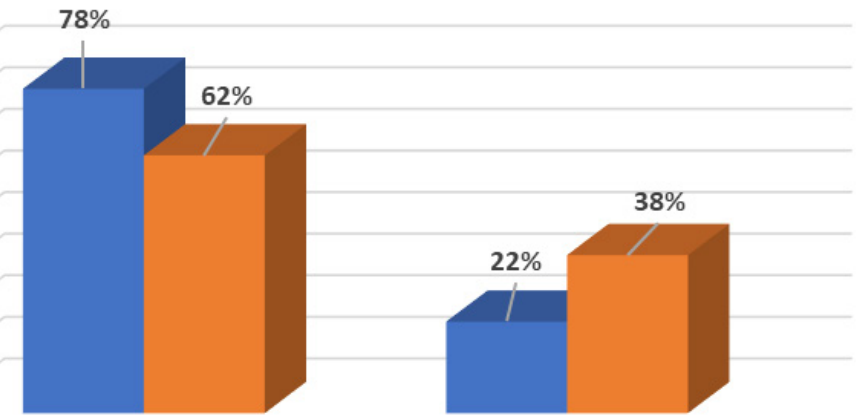

SI

NO

- Pago por tarjeta nepósito o transferencia

Otras de las acciones en materia de Responsabilidad Social, los restaurantes de la ciudad de Chilpancingo, han considerado que con la finalidad de evitar el contacto tanto de los consumidores y con monedas y papel moneda a fin de evitar la manipulación de los mismos, las empresas han optado 
por implementar el cobro de cuenta a través del pago por tarjetas y en otras ocasiones el pago a través de depósitos a cuentas o transferencias bancarias, esto sin duda resulta significativo porque se atribuye a una responsabilidad con los consumidores.

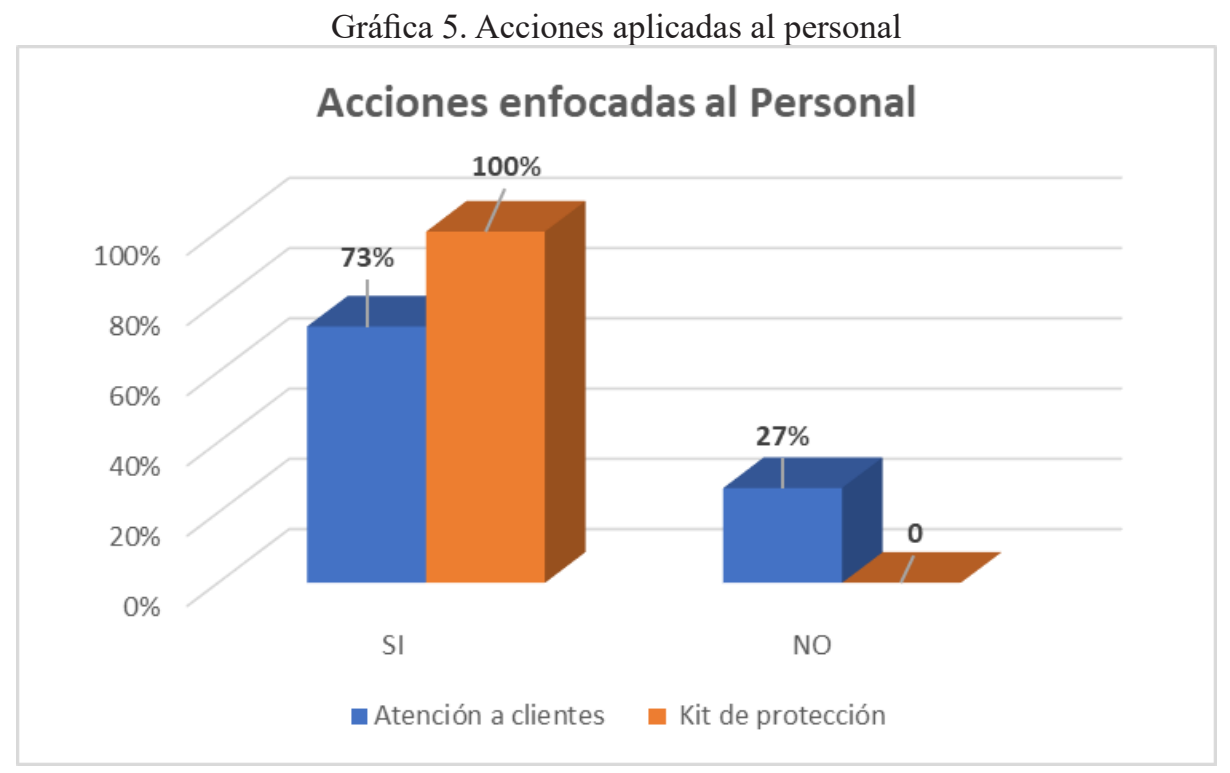

Finalmente otro elemento importante que han establecido los restaurantes tiene que ver con el personal, este elemento es muy significativo pues existe la relación con los consumidores, en este sentido el kit de protección es utilizado por el total de los restaurantes y un 73\% ha prestado a la atención a los consumidores sobre todo en el establecimiento. Lo anterior justifica parte de la Responsabilidad Social con los consumidores y empleados a fin de evitar el contacto directo cuidando los aspectos de la salud.

\section{CONCLUSIONES}

La pandemia ha venido a poner en jaque a todos los negocios a nivel mundial y en el aspecto local, también se hace evidente pues ha habido empresas de diferentes giros que han cerrado o quebrado, ello debido a que no estaban preparados antes esta situación, por otra parte tampoco pudieron reaccionar a tiempo para innovar o ser creativos en aspectos mercadológicos que les ayudara a hacer frente a esta situación mundial.

El estudio revela que los negocios de comida (restaurantes) de Chilpancingo han hecho un frente para evitar el cierre o quiebre del negocio, ello a través de la implementación de acciones de mercadotecnia, las cuales han apoyado a los restaurantes a sobrevivir ante la pandemia ocasionada por el COVID-19.

La realización de estas acciones permite a las empresas restauranteras tener una mejor imagen por parte de los consumidores, ésta es relacionada con la preocupación y el cuidado de la salud.

Dicha imagen impacta en un mejor posicionamiento lo cual impacta de manera significativa en el nivel de competitividad de las empresas restauranteras, ello al preferir por parte de los consumidores a los restaurantes que hacen uso de la Responsabilidad Social a favor de los empleados y consumidores.

Las empresas en su mayoría han implementado estrategias que son de menor costo, ello se entiende en el sentido de que la haber una crisis económica y financiera, no se cuenta con los recursos suficientes 
para poder implementarlas o desarrollarlas, sin embargo estas acciones impactan en la Responsabilidad Social con sus empleados y consumidores.

Es evidente que hace falta mayor capacitación en aspectos mercadológicos no solo para este sector económico, si no a todos los sectores, pues se pueden hacer alianzas estrategias, acciones de colaboración, generación de relaciones comerciales, entre otros, ello como estrategias empresariales que permitan de una forma colaborativa entre los diferentes negocios acotar la breca de problemáticas en torno al COVID-19.

Finalmente, también se concluye que hace falta mayores estudios, pues la propia pandemia limita realizar estudios relacionados con los consumidores, estudios longitudinales, entre otros, pues debido al riesgo de contagio, el miedo e incertidumbre limita la obtención de mayor información.

\section{REFERENCIAS}

1. ANPEC (2016). Cómo salvar de la muerte a tu tiendita. Revista Entrepreneur. Recuperado de: https://www.entrepreneur.com/article/279000

2. Auger, P., Devinney, T., Louviere, J., \& Burke, P. (2010). The importance of social product attributes in consumer purchasing decisions: a multi-country comparative study. International Business Review, 140-159.

3. Barragán, J., Guerra, P., y Villalpando, P (2017). Mercadotecnia y Responsabilidad Social Corporativa: propuesta de modelo de negocio estratégico. Daena: International Journal of Good Conscience. 12(1), 33-48. Obtenido de: http://www.spentamexico.org/v12-n1/A3.12(1)33-48.pdf

4. Chang, C. (2011). La influencia de la ética ambiental corporativa en la ventaja competitiva: el papel de mediación de la innovación verde. Journal of Business Ethics, 361-370. Recuperado de: https://link.springer.com/article/10.1007/s10551-011-0914-x

5. Cortés, F. (2019). Tendencias de Marketing 2019. MKTTotal. 1-10. Recuperado de: https://www. mercadotecniatotal.com/mercadotecnia/tendencias-de-marketing-2019/

6. Devinney, T. M., Auger, P., Eckhardt, G. y Birtchnell, T. (2006). The other CSR. Stanford Social Innovation Review, 30-37.

7. Hernández-Cuevas, F.I., Chávez-Rosado, I.G., Ulibarri-Benítez, H.A. y Morales-González, M.A. (2019). Calidad en el servicio en tiendas de abarrotes. Una perspectiva de género. Revista del Centro de Graduados e Investigación. Instituto Tecnológico de Mérida, 34(77),99-107

8. INEGI. (2020). Directorio de empresas y establecimientos. Recuperado de: https://www.inegi.org. $\mathrm{mx} /$ temas/directorio/

9. (2020). Encuesta sobre el Impacto Económico Generado por COVID-19 en las Empresas. Recuperado de: https://www.inegi.org.mx/contenidos/programas/ecovidie/2020/doc/ presentacion_ECOVIDIE.pdf

10. López, V. (2018). La competitividad de las PYMES en México: Retos y oportunidades ante un mundo globalizado. Horizontes de la Contaduría en las Ciencias Sociales, Vol.5(9). Obtenido de: https://www.uv.mx/icp/ files/2018/12/Num09-Art07-105.pdf

11. Méndez Wong, A., Mellado Siller, J. F., Reyes Ruiz, E. y Rodríguez Ramírez, K. L. (2019). La actitud de los saltillenses frente al consumo ético: Escala de Forsyth y Muncy-Vitell. Tlamati, 11(1), 24-34. Recuperado de: http://tlamati.uagro.mx/t111/t1115.pdf

12. Nielsen (2015). Estilos de Vida Generacionales. Recuperado de: https://www.nielsen.com/wpcontent/uploads/sites/3/2019/04/EstilosdeVidaGeneracionales.pdf

13. Organización Internacional de Normalización (2010) Norma ISO 26,000. Secretaría Central de ISO en Ginebra. Suiza. Obtenido de: http://www.americalatinagenera.org/ newsite/images/ U4ISO26000.pdf

14. Pineda, José. (2020): Impacto económico y social de la Covid 19 en Guerrero y los retos de la UAGro. Factores críticos y estratégicos en la interacción territorial desafíos actuales y escenarios futuros. UNAM y Asociación Mexicana de Ciencias para el Desarrollo Regional A.C, Coeditores, 
Ciudad de México. Recuperado de: http://ru.iiec.unam.mx/5236/1/4-217-Pineda.pdf

15. Ramírez Martínez M.A. (2021). Marketing Digital Como Estrategia de las Empresas de Alimentos de Villahemosa, Tabasco Frente al COVID-19. European Scientific Journal, ESJ, 17(4), 12. https:// doi.org/10.19044/esj.2021.v17n4p12

16. Rodríguez, E., Carmona, A., \& Bautista, L. (2017). Análisis de Condiciones de Cierre de Mipymes Comerciales en la Zona Conurbada Zacatecas-Guadalupe en México. Revista Global de Negocios. Volumen 5, número 7. Recuperado de: https://www.theibfr.com/es/download/rgn/2017-rgn/rgn v5n7_2017/RGN-V5N7-2017.pdf

17. Secchi, D. (2007). Teorías utilitarias, gerenciales y relacionales de la responsabilidad social corporativa. International Journal of Managemwent reviews. 9(4), 347. Recuperado de: https:// doi.org/10.1111/j.1468-2370.2007.00215.x

18. Susano García, J. L., Almazán Adame, A. A., Cabrera Ríos, M. del S. y Méndez Wong, A. (2020). La responsabilidad social en restaurantes de Chilpancingo de los Bravo, Guerrero, México. Una visión como estrategia de marketing. Tlamati, 11(1), 41-46.

19. Stanley, M. G. (2014). Responsabilidad Social y su impacto en las decisiones de marketing. Decimonovenas Jornadas "Investigaciones en la Facultad de Ciencias Económicas y Estadística". Universidad Nacional de Rosario. Obtenido de: https://rephip.unr.edu. ar/bitstream/ handle/2133/7441/Stanley\%2C\%20etica\%20y\%20rse\% 20marketing. pdf? sequence $=3 \&$ isAllowed $=y$

20. Susano García L.J., Bonilla Gómez A.M. \& Cabreara Ríos M. S. (2021). Implementación de Estrategias de Marketing en Tiendas de Abarrotes para Hacer Frente al COVID-19 en Guerrero, México. European Scientific Journal, ESJ, 17(4), 213. https://doi.org/10.19044/esj.2021. v17n4p213

21. Willer, H. y Lernoud, J. (2016). The World of Organic Agriculture. Statistics and Emerging Trends 2016. Research Institute of Organic Agriculture FiBL and IFOAM Organics International. Obtenido de: http:// orgprints.org/31151/1/willerlernoud-2016-world-oforganic.pdf 